\title{
Root-soil contact dynamics of Vicia faba in sand
}

\author{
Nicolai Koebernick (D) - Steffen Schlüter • Sebastian R. G. A. Blaser • \\ Doris Vetterlein
}

Received: 1 March 2018 / Accepted: 26 July 2018 / Published online: 14 August 2018

(C) The Author(s) 2018

\begin{abstract}
Aims Root shrinkage in drying soil has been shown repeatedly. The aim of this study was to investigate the dynamics of root-soil contact and its relationship with plant water status during soil drying.

Methods The development of root-soil contact of Vicia faba L. during a drying period was studied. Plants $(N=4)$ were grown in cylinders filled with a sandy soil. Samples were repeatedly scanned with an X-ray CT scanner to visualize root-soil contact. Soil matric potential, transpiration rate, and stomatal conductance were measured daily. Results Root-soil contact was lower in taproots than in lateral roots at any time. Transpiration rate and stomatal
\end{abstract}

Responsible Editor: Anton Wasson

Electronic supplementary material The online version of this article (https://doi.org/10.1007/s11104-018-3769-4) contains supplementary material, which is available to authorized users.

N. Koebernick $(\bowtie)$

Bioengineering Sciences Research Group, Engineering Sciences Academic Unit, Faculty of Engineering and the Environment, University of Southampton, University Road, Southampton SO17 1BJ, UK

e-mail: n.koebernick@soton.ac.uk

N. Koebernick · S. Schlüter · S. R. G. A. Blaser •

D. Vetterlein

Department of Soil Physics, Helmholtz Center for Environmental Research, Theodor Lieser Straße 4, 06120 Halle (Saale), Germany

N. Koebernick · D. Vetterlein

Insitute of Agricultural and Nutritional Sciences,

Martin-Luther-Universität Halle-Wittenberg,

Von-Seckendorff-Platz 3, 06120 Halle (Saale), Germany conductance decreased before roots started to shrink. Root-soil contact decreased significantly over the course of the drying period, starting at soil matric potentials below $-20 \mathrm{kPa}$. Root shrinkage did not differ significantly between taproots and laterals.

Conclusions This study confirms previous findings with Lupinus albus roots in that roots shrink after transpiration rate decreases. The dynamics of root shrinkage are governed by soil water availability and transpirational demand.

Keywords Root-soil contact · X-ray CT · Rhizosphere · Drought stress $\cdot$ Root shrinkage

\section{Introduction}

Root-soil contact (RSC) is critical for plant-soil interactions. Complete RSC can obstruct the diffusion of gasses, while low contact can limit the uptake of water and solutes by constraining the hydraulic pathway (Veen et al. 1992). The degree of RSC is influenced by the texture and the water status of the growing medium (Herkelrath et al. 1977; Kooistra et al. 1992; Tinker 1976). As roots advance in the soil, they penetrate and deform the soil matrix and this can lead to appreciable compaction around the roots (Bruand et al. 1996; Vollsnes et al. 2010). Based on density measurements around roots and inflatable tubes, Dexter (1987) formulated a simple model of soil compression around roots. Soil density increases exponentially towards the root surface, as the volume occupied by the expanding root 
is accommodated by an equivalent loss of porosity. Hettiaratchi et al. (1990) developed a punch indentation model for root growth showing that cyclic changes of radial and axial root expansion may facilitate penetration of compacted soils.

Reduced RSC occurs when roots grow into macropores or loose soil where they retain only partial contact or as a consequence of root shrinkage (Tinker 1976). Living root tissues shrink when they are dried beyond a certain threshold. Theoretical considerations about the formation of air gaps adjacent to the root surface date back to the 1950s (Bernstein et al. 1959; Philip 1957). Direct evidence of shrinking roots in soil was provided by Huck et al. (1970), who observed diurnal diameter changes by $25 \%$ of a cotton root segment in response to changes in transpirational demand. Cole and Alston (1974) determined that shrinkage occurs at a water potential between -0.5 and $1 \mathrm{MPa}$ in excised maize roots. The first study to analyze root shrinkage with actual measurements of RSC was conducted by North and Nobel (1997a). They determined RSC of droughted Agave deserti roots with a thin-section technique developed by van Noordwijk et al. (1992). They found that RSC had decreased from 94 to $21 \%$ after 14 days of drought. In a second treatment, they re-established RSC of the droughted roots by vibrating the samples. This resulted in more negative soil water potentials around the roots, indicating higher root water uptake and hence a limiting role of RSC in water uptake. The vibrated roots also shrank less, which suggests a feedback mechanism between contact loss and root shrinkage. Such a mechanism was proposed by Carminati et al. (2013), who stated that once a gap is formed at the root surface, the additional resistance to water flow will lead to increased root shrinkage and thus shrinkage is a selfenforcing process. The reliance on destructive methods has hindered researchers to study in detail the temporal dynamics of root shrinkage and gap formation with regard to plant and soil water status.

Recent advances in non-invasive imaging techniques allow the direct observation of the root-soil interface during plant growth. Particularly X-ray computed tomography (X-ray CT) is now widely used in the study of plant-soil interactions (Mooney et al. 2012). While Haling et al. (2013) found that X-ray CT significantly accelerated the decay of excised Vicia faba roots in soil, Zappala et al. (2013) suggested that careful choice of scanning parameters enables repeated scanning of roots without impacting root architecture. Repeated scanning allows the observation of dynamic changes of root-soil interactions with time and following the drying of soil. Carminati et al. $(2009,2013)$ first observed the formation of air gaps with X-ray CT. In their experiments using Lupinus albus roots in a sandy soil substrate, air gaps appeared at soil matric potentials between -10 and $-20 \mathrm{kPa}$, after transpiration rate of the plants started to decrease. They concluded that air gaps were not the cause but rather a consequence of water stress. The authors compared the shrinkage of taproots with lateral roots and found that taproots shrank to a higher degree than laterals. Such a dissimilar behaviour raises the question, if the observed differences are merely a result of the larger diameter of taproots or if such functional plasticity is a plant strategy for the efficient utilization of resources, as it was proposed by Carminati and Vetterlein (2013). MRI studies on pine roots have shown that the taproot can play a major role in water uptake, sometimes exceeding lateral roots in uptake efficiency (MacFall et al. 1991). If taproots shrink more than laterals, this higher uptake efficiency may only be sustained when there is good RSC. In Carminati et al. (2013) the detection of air gaps at lateral roots was complicated by limited resolution and no method for the determination of RSC was available. Therefore the possibility of partial contact was omitted, while it may play an important role in maintaining water and nutrient uptake at low water potentials. Tinker (1976) argued that, due to surface tension forces, it is more likely that shrinking roots lose contact on one side of their surface, while the other side remains in contact with soil. $\mathrm{Nu}-$ merical simulations by Nye (1994) showed that partial contact loss may lead to strong gradients of water potential within the root cortex. Simulated water potential was highest in the part of the cortex that was near the contact zone with soil and steeply decreased away from it. Thus, when a root starts to lose contact at one point of its surface, further shrinkage is more likely to occur near this point and shrinkage will be eccentric.

To analyze the extent of root shrinkage and the occurrence of partial contact, a method for the quantification of RSC is needed. A robust method of RSC determination in X-ray CT images has been developed by Schmidt et al. (2012). After segmenting the images into root soil phase they used a morphological dilation operation on the soil phase so that it overlapped with the root surface. Basic set theory then provided the degree of RSC. In the present paper, we present an alternative method of RSC determination by directly deriving it from the surface area of roots, pores and soil matrix. 
The purpose of this study is to extend previous experiments of Carminati et al. (2013) on the dynamics of root shrinkage with Lupinus albus by (i) including a method for RSC quantification, (ii) using a different species, Vicia faba L., and (iii) imaging at a higher resolution for a better comparison of taproots and laterals. The use of Vicia faba was partly motivated by the comparatively large diameter of its roots, in particular its laterals, which will simplify the detection of gaps at the surface of lateral roots. The use of a different species may also help to generalize previous findings.

\section{Materials and methods}

Plant material and experimental setup

The experiment was set up with four replications (Vicia I, II, III, IV). PVC cylinders (8.1-cm diameter, $20-\mathrm{cm}$ height) were filled with a sandy soil from the field site "Hühnerwasser", which consisted of $92 \%$ sand, $5 \%$ silt, and $3 \%$ clay. The soil was sieved to $<2 \mathrm{~mm}$ and packed into the cylinders by passing it through two sieves. The procedure resulted in a homogeneous packing and a bulk density of $1.62 \pm 0.007 \mathrm{Mg} \mathrm{m}^{-3}$ (porosity = $38.9 \%$ ). Seeds of Vicia faba L. cv. Fuego were surface sterilized in $10 \% \mathrm{H}_{2} \mathrm{O}_{2}$ for $10 \mathrm{~min}$ and soaked for one hour in a saturated $\mathrm{CaSO}_{4}$ solution. Seeds were placed on wet filter paper and stored in a dark cabinet at room temperature for $24 \mathrm{~h}$ to allow for germination. Germinated seeds were carefully transplanted in the soil at $1 \mathrm{~cm}$ depth. The soil surface was covered with quartz gravel $(2-5 \mathrm{~mm})$ to minimize evaporation. Liquid flower fertilizer (Terrasan $\mathrm{GmbH}$, containing $7 \% \mathrm{~N}, 1.3 \% \mathrm{P}$, $5 \% \mathrm{~K}$ ) was diluted 1:100 and $100 \mathrm{ml}$ per column of the solution was applied to plastic reservoirs, the cylinders were placed in these reservoirs. After all the water was taken up by capillary rise, an additional $50 \mathrm{ml}$ of deionized water was applied. Free water was always available in the reservoirs to provide well-watered growth conditions for 14 days $\left(\psi_{m}=0 \mathrm{kPa}\right.$ at the bottom of the columns). At the start of the drying period the reservoirs were removed. The drying period lasted 13-23 days varying between different samples. At the end of the experiment plants were rewatered by placing the plastic reservoirs with $100 \mathrm{ml}$ deionized water at the bottom of the samples for three hours. Plants were grown in a climate chamber under controlled conditions (14 h photoperiod, photon flux density $350 \mathrm{mmol} \mathrm{m}^{-2} \mathrm{~s}^{-1}$, constant temperature $23{ }^{\circ} \mathrm{C}, 65 \%$ relative humidity). Lateral roots touched the sides of the pots after approx. 7 days and the root system became pot bound after 2 weeks of growth. Throughout the experiment the columns were placed on balances and the weight was continuously recorded. From the balance data, total plant transpiration was calculated, ignoring weight gain through plant growth. Relative transpiration rate was calculated as the ratio between actual transpiration rate and the maximum transpiration rate at the start of the drying period. Microtensiometers (Vetterlein et al. 1993) were inserted at 5-cm depth and soil matric potential was measured at a $10 \mathrm{~min}$ interval. Stomatal conductance was measured daily using a steady-state leaf porometer (Decagon Devices, Version 6.0) on the abaxial side of the youngest unfolded leaves. A measurement was made on each leaflet and the mean of two measurements was recorded.

\section{CT scanning}

CT scans of the samples were performed with an industrial X-ray micro-CT scanner (X-Tek HMX 225) equipped with a finefocus X-ray tube (spot size $5 \mu \mathrm{m}$ ) and a $512 \times 512$ detector panel. Initial scans of the entire columns were performed to locate the taproot and laterals. Based on these scans, a region of interest (ROI) with a field of view $3 \times 3 \mathrm{~cm}, 60 \mu \mathrm{m}$ voxel side length was chosen for ROI scanning, so that spatial details $<120 \mu \mathrm{m}$ can be detected robustly. The region of interest was located at a depth of 3-6 cm below the soil surface in the center of the cylinder in all the samples. This choice was based on the absence of major macropores and the presence of taproot and laterals. The soil depth was chosen in order to analyse well established roots, where less change of root architecture over time was expected. ROI scanning, where the field of view is smaller than the total sample size, can be used to image a subsample of the entire object at a higher resolution. A precondition for ROI scanning is the macroscopic homogeneity of the region outside the field of view, which is satisfied for homogeneously packed soil columns. A downside of this method is the occurrence of truncation artefacts, most commonly appearing as a bright concentric band at the truncation edges. Local CT scans were recorded with X-ray energy of $130 \mathrm{kV}$ and a current of $500 \mu \mathrm{A} .800$ projections with an exposure time of $400 \mathrm{~ms}$ using 2 frames per projection were recorded. The resulting total scan time was $10.6 \mathrm{~min}$. X-ray dose 
per scan was estimated using the free online calculator RadProDose (McGinnis 2002-2006). The estimated dose was 1.1 Gy per scan. With a maximum number of 10 scans per sample, the cumulative dose was still well below the critical dose ( $\sim 30 \mathrm{~Gy})$ to influence plant growth (Zappala et al. 2013). The samples were not scanned on a daily basis to reduce the number of total scans per sample. Instead, CT scanning was performed at values of soil matric potential where root shrinkage was observed in previous experiments with Lupinus albus in the same substrate (Carminati et al. 2013). Scan dates are recorded in Table 1. CT scans were performed in the middle of the day phase of the $14 \mathrm{~h}$ photoperiod to remove diurnal influences. When the plants were rewatered, additional CT scans were performed three hours after rewatering. These scans served as a rescue treatment and will be referred to as rewatered controls.

Image processing and analysis

Image analysis was restricted to a cylinder of 400 pixels $(2.4 \mathrm{~cm})$ diameter to remove truncation artefacts caused by the ROI scanning. Cone beam artefacts at the top and the bottom of the image were removed by cropping, reducing the height of the images to $2.7 \mathrm{~cm}$ (450 pixels). Raw images (Fig. 1a) were filtered using an adaptive Gaussian filter to remove small scale noise while preserving edges (Fig. 1b). An unsharp mask (Schlüter et al. 2014) was performed subsequently to enhance the contrast at the edges of objects (Fig. 1c). The histograms of the resulting images were bimodal and despite significant overlap showed distinctive peaks for the root phase and the solid soil phase (see supplementray material, Fig. S1). Due to its small volume and partial volume effects, the air phase had no separate peak but was represented by a tail at the lower end of the histogram. Therefore, a stepwise procedure was applied to segment the three different phases. In the first step, the root phase including air was segmented from the soil phase. This threshold $\mathrm{T}_{\text {root }}$ was computed by using the average threshold of five standard histogram-based thresholding methods following the procedure described in Schlüter et al. (2014). Disconnected air filled pores were excluded by applying region growing from a seed point within the root system and a subsequent opening operation. In the second step, the histogram of the resulting region, which contained the root system and the air gap, was used to find the threshold $\mathrm{T}_{\text {air }}$ between air and roots with Rosin's method for unimodal thresholding (Rosin 2001).

The segmented images contained artefacts caused by partial volume effects at the transition between air and soil phase (Fig. 2b). These voxels were erroneously classified as root voxels. A procedure to remove these partial volumes by marking all root voxels with both air and soil voxels in their neighbourhood as transition voxels was developed (Fig. 2c). Transition voxels were reclassified as either soil or air voxels, based on the comparison of their grey value with the mean grey value of the soil phase and the air phase, respectively (Fig. 2d).

The surface area of the different phases was quantified directly on binary images by computing the second Minkowski functional $\mathrm{M}_{1}$ of each phase (for further detail, see Vogel et al. 2010). Therefore the images have to be segmented into structure and background for each phase that is evaluated. The surface area A of the evaluated phase does not yield the root-soil boundary directly but is the sum of the boundary areas with both remaining phases. Calculation of $\mathrm{A}$ of each individual phase yields the linear system

$$
\begin{aligned}
A_{\text {root }} & =A_{\text {root-air }}+A_{\text {root-soil }} \\
A_{\text {soil }} & =A_{\text {root-soil }}+A_{\text {air-soil }} \\
A_{\text {air }} & =A_{\text {root-air }}+A_{\text {air-soil }},
\end{aligned}
$$

which is solved for RSC area

$A_{\text {root-soil }}=\frac{A_{\text {root }}+A_{\text {soil }}-A_{\text {air }}}{2}$

Percentage of RSC is then simply calculated by

$\rho=\frac{A_{\text {root-soil }}}{A_{\text {root }}} \times 100$

For the analysis of root soil contact, taproot and laterals were analyzed separately. They could be easily separated by their different diameters. The opening map of the root phase was calculated with a maximum inscribed sphere method and subsequently segmented by using a threshold which excluded lateral roots.

First order lateral roots were disconnected from the taproot by this step. Second order lateral roots were not separated but subsumed to the first order lateral they originated from. Due to the large variability in growth dynamics between the four biological replicates, a subsampling routine was developed, which enabled the analysis of RSC for individual first order lateral roots within each CT scan. Lateral roots were subsampled 
Table 1 Overview of scanned sample replicates, showing the number of first order lateral roots in the region of interest (ROI) and the time of CT scans for different samples. Soil matric potentials at the time of the scans are given in parentheses

\begin{tabular}{|c|c|c|c|c|c|c|c|c|}
\hline \multirow[b]{2}{*}{ Vicia I } & \multirow{2}{*}{$\frac{\text { \# of laterals in ROI }}{20}$} & \multicolumn{7}{|c|}{ Time of CT scans after start of drought (soil matric potential [hPa]) } \\
\hline & & $\begin{array}{l}8 \mathrm{~d} \\
(-125)\end{array}$ & $\begin{array}{l}11 \mathrm{~d} \\
(-250)\end{array}$ & $\begin{array}{l}12 \mathrm{~d} \\
(-522)\end{array}$ & $\begin{array}{l}13 \mathrm{~d} \\
(<-550)\end{array}$ & $13 \mathrm{~d}$ (rewater) & & \\
\hline Vicia II & 24 & $\begin{array}{l}12 \mathrm{~d} \\
(-84)\end{array}$ & $\begin{array}{l}13 d \\
(-122)\end{array}$ & $\begin{array}{l}14 d \\
(-199)\end{array}$ & $\begin{array}{l}15 d \\
(-335)\end{array}$ & $\begin{array}{l}16 \mathrm{~d} \\
(-444)\end{array}$ & $\begin{array}{l}17 d \\
(-461)\end{array}$ & $\begin{array}{l}17 \mathrm{~d} \\
\text { (rewater) }\end{array}$ \\
\hline Vicia III & 16 & $\begin{array}{l}13 \mathrm{~d} \\
(-64)\end{array}$ & $\begin{array}{l}18 \mathrm{~d} \\
(-107)\end{array}$ & $\begin{array}{l}20 \mathrm{~d} \\
(-211)\end{array}$ & $\begin{array}{l}21 \mathrm{~d} \\
(-300)\end{array}$ & $\begin{array}{l}22 \mathrm{~d} \\
(-332)\end{array}$ & $\begin{array}{l}23 \mathrm{~d} \\
(-377)\end{array}$ & $\begin{array}{l}23 \mathrm{~d} \\
\text { (rewater) }\end{array}$ \\
\hline Vicia IV & 6 & $\begin{array}{l}11 \mathrm{~d} \\
(-73)\end{array}$ & $\begin{array}{l}16 \mathrm{~d} \\
(-151)\end{array}$ & $\begin{array}{l}20 \mathrm{~d} \\
(-387)\end{array}$ & $\begin{array}{l}21 \mathrm{~d} \\
(-446)\end{array}$ & $\begin{array}{l}22 \mathrm{~d} \\
(-469)\end{array}$ & $\begin{array}{l}23 \mathrm{~d} \\
(<-550)\end{array}$ & $\begin{array}{l}23 \mathrm{~d} \\
\text { (rewater) }\end{array}$ \\
\hline
\end{tabular}

using a labelling algorithm, which assigned a label number to each individual first order lateral root. The number of lateral roots present in the ROI was between 6 and 24 (Table 1). That is, while the sample number for tap roots is $n=4$, the number of technical replicates for lateral roots is much larger. Root soil contact was calculated for the taproot, all lateral roots, and each individual lateral root separately. To this end, each individually labelled root was opened separately and dilated by 2 voxels. The dilated structure was saved as a mask (see Fig. 2d). Computation of root-soil contact for individual roots took only the boundary areas present within the mask into account. It has to be noted that the dilation step meant that the mask representing the taproot contained the basal part of emerging lateral roots.

$\mathrm{RSC}$ is here defined as the proportion of the root surface in contact with the soil matrix. Root hairs are excluded from this definition. Typical root hair diameters are between 6 and $16 \mu \mathrm{m}$ (Leitner et al. 2010), which is too small to be captured both precisely and representatively using current industrial CT scanners. An alternative way of calculating root soil contact is provided by morphology and basic set theory (Schmidt et al. 2012): Dilation of the root phase yields all the
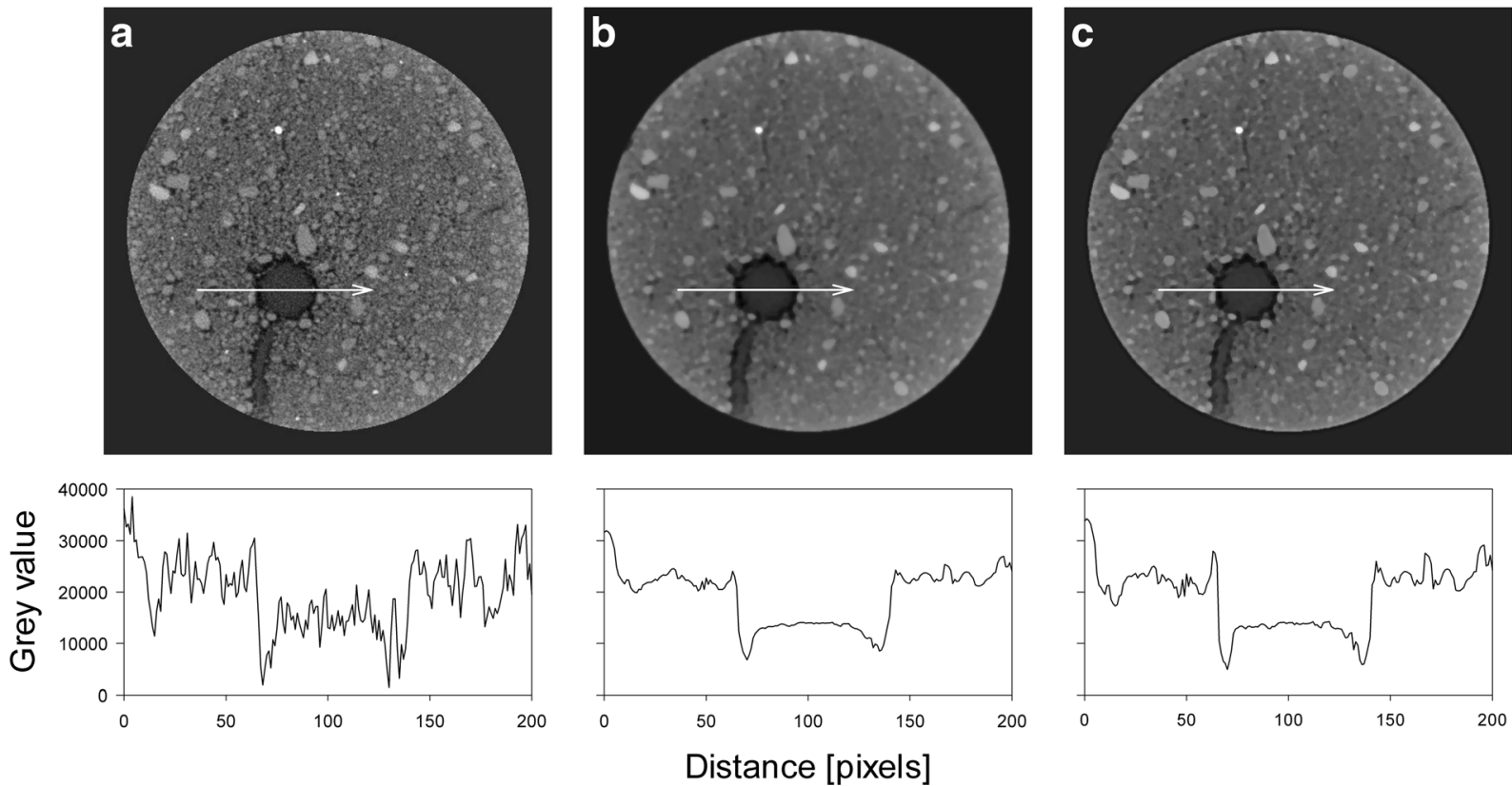

Fig. 1 Horizontal slice of a region of interest tomography of Vicia I at Day 13 of the drying period, showing the effect of image processing. Below, the profile of grey values along the white arrows in the images containing soil, the taproot, and surrounding

air gaps is shown (a) raw image (b) filtered with adaptive gaussian filter (c) sharpened with unsharp mask. Arrows have a length of 200 pixels, corresponding to $12 \mathrm{~mm}$ 


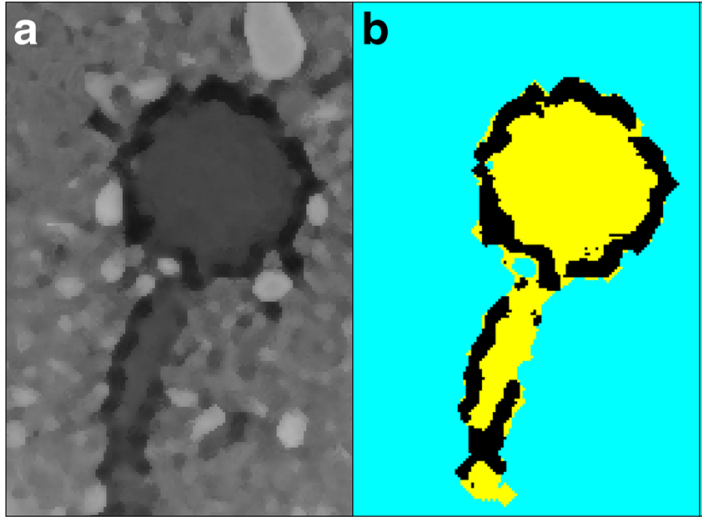

Fig. 2 Enlarged cutout of the horizontal slice seen in Fig. 1, showing the results of segmentation and post-processing. a Filtered grey scale image. b Classified image after segmentation. Soil phase is cyan, root phase is yellow, and air phase is black. Disconnected air-filled pores are part of the soil phase. c Transition voxels between soil and air phase are marked in red. Note that

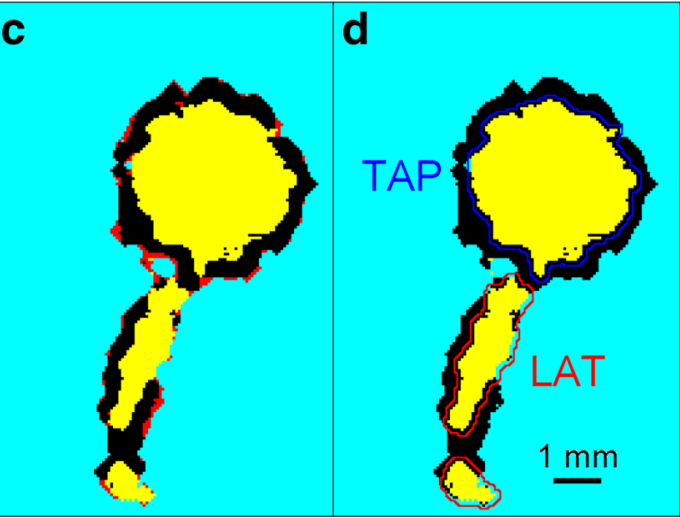

transition voxels were located based on 3D images. d Classified image after reclassification of transition voxels. The masks for the calculation of root-soil contact in individual roots are indicated in blue for taproot (TAP) and red for the lateral root (LAT), respectively voxels at the root interface. A subsequent intersection with the soil phase yields only soil voxels in contact with the root. The ratio of both volumes is calculated to estimate RSC. This is possible, because the ratios of the volume and the respective area of a defined shape are the same. To validate our direct method for RSC quantification, RSC was determined with the alternative method based on the dilation of the root phase and subsequent intersection with the soil phase. The two methods showed good agreement (see supplementary material Fig. S2).

The effect of the choice of the threshold on the results was tested by perturbing the original $\mathrm{T}_{\text {air }}$ by segmenting and reanalyzing the images with a manually changed $\mathrm{T}_{\text {air }}$. A 5\% higher and a 5\% lower $\mathrm{T}_{\text {air }}$ was chosen, thus creating an envelope of $10 \%$ around the computed threshold. The difference of RSC due to the variation of $\mathrm{T}_{\text {air }}$ by $10 \%$ was on average $11.5 \pm 4.7 \%$.

Image analysis was performed using the freely available software ImageJ and QuantIm (www.quantim.ufz. de). Statistical analysis was performed in SPSS 22 (IBM Corp, Armonk, NY). Rewatered control treatments were subjected to Kruskall-Wallis-Test to test for significant differences between plants. Friedman's test was applied to test for statistical differences between repeated measurements of each individual plant replicate. Post hoc analysis with Wilcoxon signed-rank tests was conducted with a Bonferroni correction applied, comparing each repeated measurement to the rewatered control.

\section{Results}

Effect of soil drying on plant transpiration

The duration of the drying period varied between the four replications. In Vicia I, which was the largest plant, the soil dried faster than in the remaining samples (Fig. 3), the sample was rewatered after 13 days. Vicia II was rewatered after 17 days of drying. The remaining samples Vicia III and IV had lower transpiration and were rewatered on Day 23. Soil matric potential after rewatering could not be measured because air bubbles had formed in the tensiometers at the final stage of drying.

Stomatal conductance of the youngest unfolded leaves decreased rapidly after the start of the drying period (Fig. 4a). At soil matric potentials below $-5 \mathrm{kPa}$ stomatal conductance was strongly reduced. Further drying of the soil only slightly affected stomatal conductance. $24 \mathrm{~h}$ after rewatering the recovery of stomatal conductance was measured. Moderate recovery was seen in Vicia I $\left(100.3 \mathrm{mmol} \mathrm{m}^{-2} \mathrm{~s}^{-1}\right)$ and Vicia II $\left(66.7 \mathrm{mmol} \mathrm{m}^{-2} \mathrm{~s}^{-1}\right)$, while in Vicia III and IV there was no recovery after $24 \mathrm{~h}\left(1.2\right.$ and $4.4 \mathrm{mmol} \mathrm{m}^{-2} \mathrm{~s}^{-1}$, respectively). Reduction of stomatal conductance was accompanied by a reduction of relative transpiration rate (Fig. 4a, b). Maximum transpiration rate was observed on Day 4 of the drying period in Vicia III and on Day 2 for the remaining samples. Between -5 to $-10 \mathrm{kPa}$, 
Fig. 3 Development of soil matric potential over time during the drying period. Values are the mean soil matric potential of one day. Different symbols indicate individual replicates

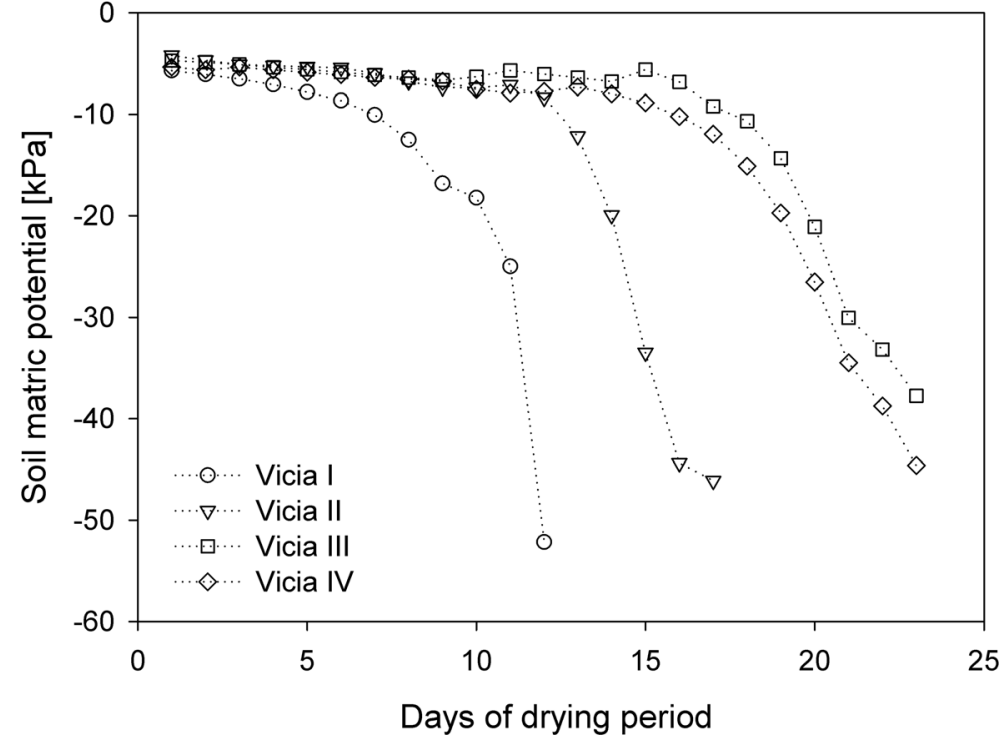

transpiration decreased to about half of the maximum, below $-10 \mathrm{kPa}$ relative transpiration still decreased, but the reduction was less steep. Minimal relative transpiration showed high variability between different samples, ranging from $1 \%$ in Vicia IV to $31 \%$ in Vicia III.

\section{Development of RSC}

The different phases, soil, root, and air could be distinguished according to their grey values. In Fig. 2a coarse sand particles appear bright, the soil matrix appears light grey, roots are dark grey, and air filled spaces appear black. Due to the limited resolution $(60 \mu \mathrm{m})$, water filled

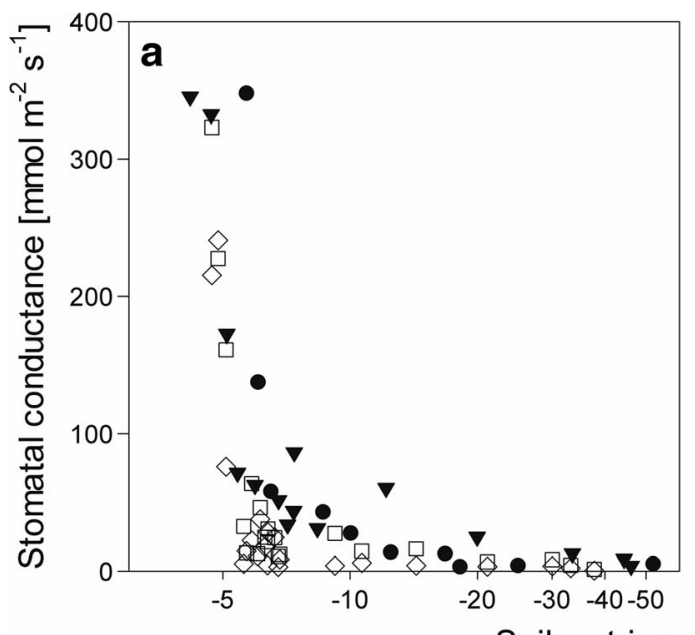

pores could not be distinguished from the soil matrix and detectable air filled pore space was limited to macropores $>120 \mu \mathrm{m}$ diameter. The temporal development of the three phases from the start of the drying period (left column), to the end of the drying period (center) up to $3 \mathrm{~h}$ after re-watering (right column) is depicted in Fig. 5. All regions of interest contained taproots and laterals. Samples I-III had similar architectures, while sample IV differed in that (i) there were overall fewer roots in the analyzed volume, and (ii) only the upper 9-mm of the taproot could be clearly distinguished. Further down, the root divided into three roots of similar diameter (Fig. 5j-1). These were classified as

Soil matric potential $[\mathrm{kPa}]$

Fig. 4 Development of stomatal conductance (a), and relative transpiration rate (b) over soil matric potential. Note the logarithmic scaling of soil matric potential. Different symbols indicate individual replicates 
Fig. 5 3D rendered view of the root systems (grey) and air gaps adjacent to the root (red) within the region of interest at the first scan (left column), before rewatering (middle column), and $3 \mathrm{~h}$ after rewatering (right column). (a-c) Vicia I, (d-f) Vicia II, (g-i) Vicia III, (j-1) Vicia IV.

Dimensions of the shown region are $2.7 \mathrm{~cm} \times 2.4 \mathrm{~cm} \times 2.4 \mathrm{~cm}$. The detached root fragment seen at the top in a and c was not detected in b
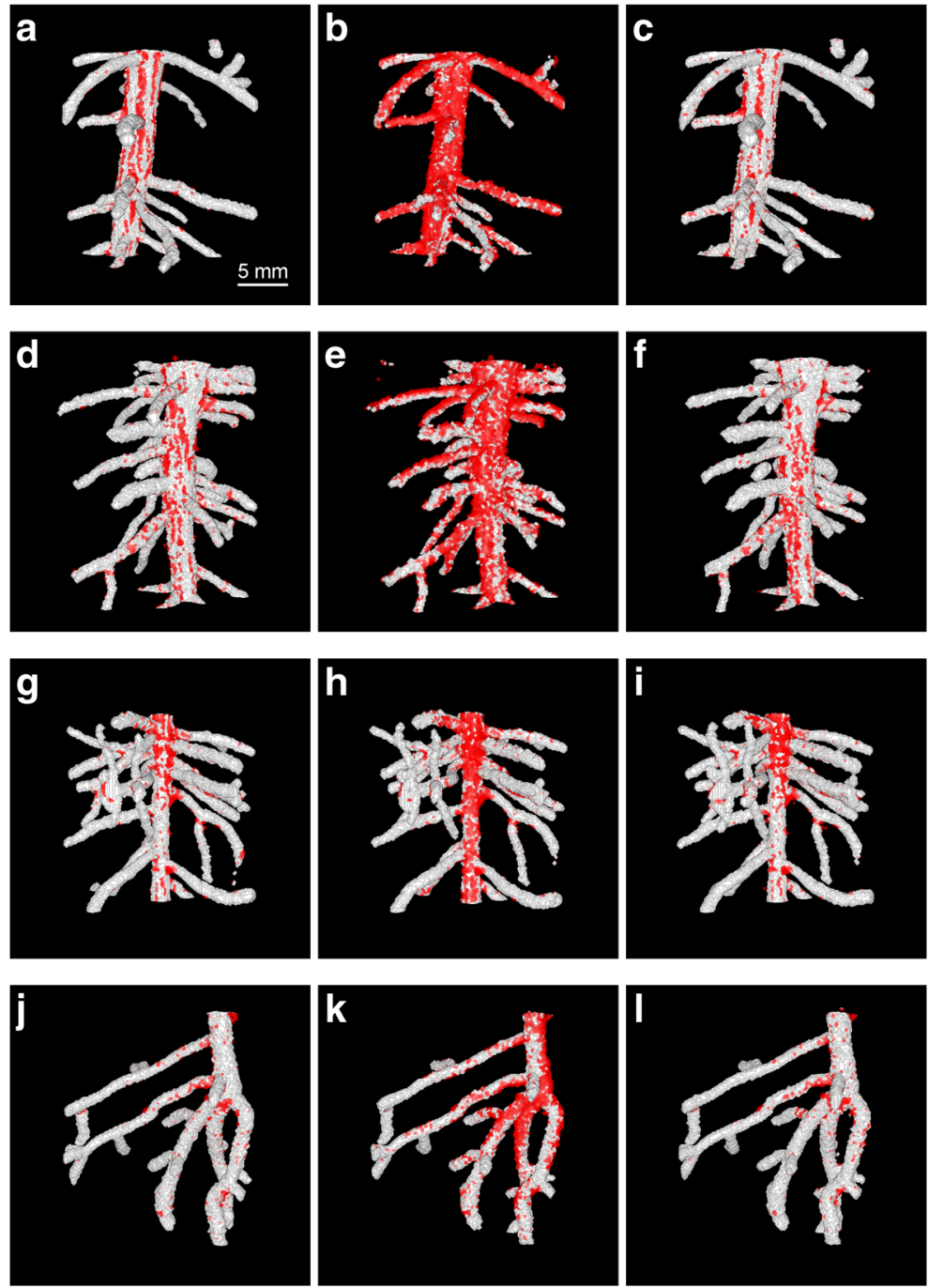

lateral roots. There were no marked differences in root architecture over time within the analyzed region of interest. The maximum change of the combined volume of roots and air within the analyzed region of interest was on average $7.4 \pm 1.6 \%$ over the course of the experiment (Fig. 6), indicating that (i) the segmentation procedure was relatively robust; (ii) the volume of the soil phase did not change significantly during soil drying; and (iii) there was no significant root growth within the scanned region. In some instances, a part of the root system was not successfully segmented at a specific time step, which partly explains slight changes of the combined volumes (Fig. 6). The single volumes of the root and the air phase changed markedly over the drying period. A decrease of root volume was accompanied by an increase of air volume, signifying the shrinkage of roots. There was a considerable variation between the different samples in both the extent and the dynamics of shrinkage. Vicia I and II showed more shrinkage than the remaining samples III and IV. At the end of the drying period, root volume of both Vicia I and Vicia II was reduced to $71 \%$ of the respective original volume at the first scan. Root volume of Vicia IV at the end of the drying period was $79 \%$ of the original volume. Roots of Vicia III shrank to a lesser extent; the final volume was $89 \%$ of the original volume. Three hours after rewatering, root volume increased in all samples, while air volume decreased simultaneously. Temporal dynamics of root shrinkage showed a high variation between different samples. Initiation of root shrinkage was apparent on Day 11 of the drying cycle in Vicia I, on Day 16 in Vicia II, while in Vicia III and IV it started only on 


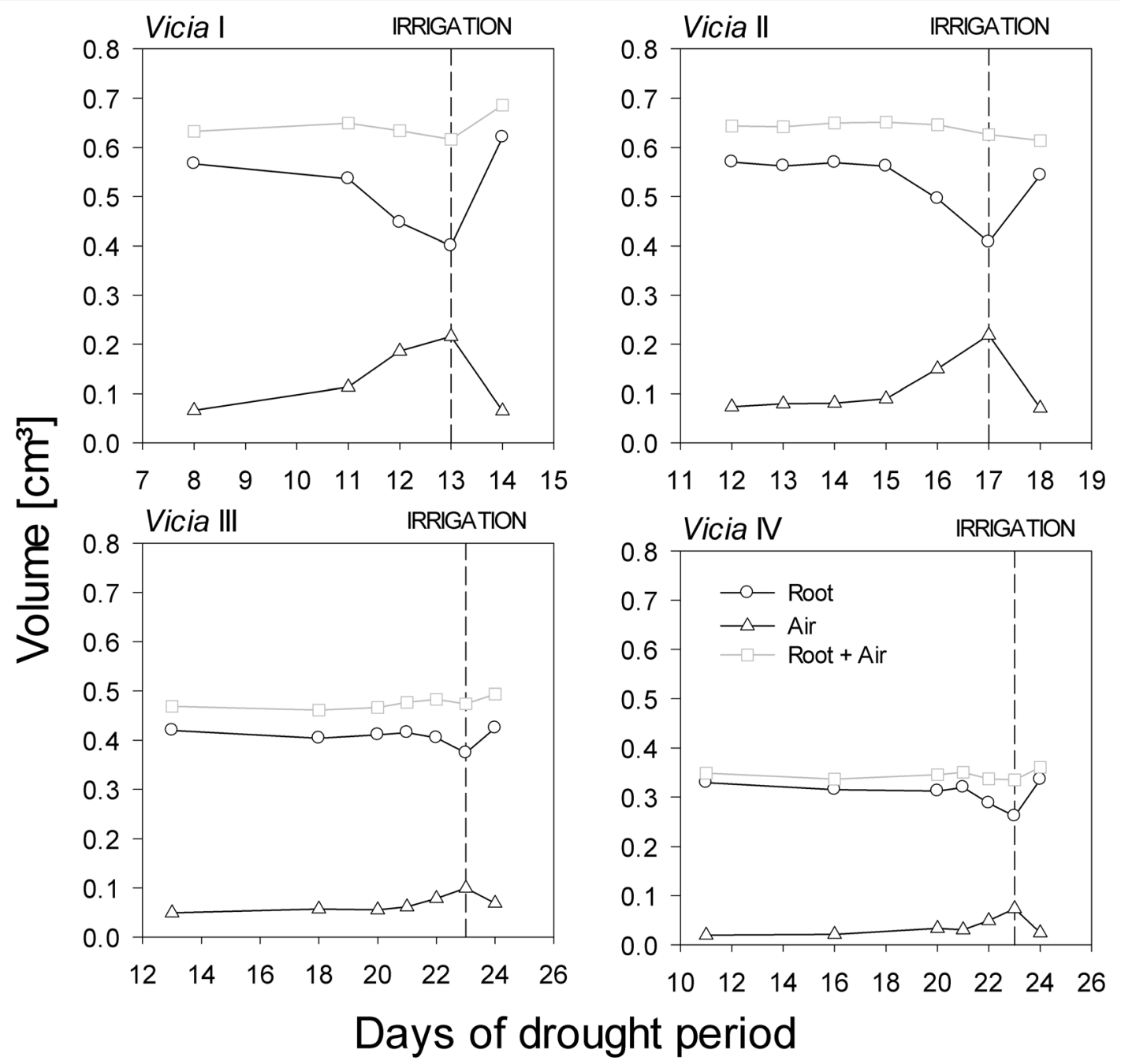

Fig. 6 Development of volume of the root and air phase within the region of interest over time, respectively. Black triangles represent air volume, black circles represent root volume. Grey

Day 23. Only in Vicia I, shrinkage was observed between the first two scans. In the remaining samples both air and root volume changed only slightly until Day 16 (Vicia I) and Day 22 (Vicia III and IV), when root shrinkage was initiated. The shape of the volume changes over time showed an increasing rate of shrinkage with the duration of the drying period (Fig. 6).

For further analysis taproot and laterals were analyzed separately. Among laterals, each lateral was analysed separately to compensate for the large variability among the four biological replicates (Vicia I to Vicia IV) (Figs. 7 and 8). Relative root shrinkage was calculated in relation to the root volume of the rewatered control. Maximum volumetric shrinkage at the driest squares represent the combined volume of both phases. Note the different ranges of the time axis

point was $23.5 \pm 3.9 \%$ for taproots and $27.2 \pm 13.8 \%$ for laterals. There was no statistically significant difference between taproots and laterals (Mann-Whitney $\mathrm{U}=$ $6, p=0.69$ ).

A major difference between taproot and laterals was observed when analyzing RSC per se. In the rewatered controls, average RSC of lateral roots was $84.1 \pm 1.0 \%$, compared to RSC of only $42.1 \pm 0.6 \%$ of taproots (Fig. 7). Taproot RSC was consistently much smaller than lateral root RSC over the whole duration of the drying period. Subsampling of individual lateral roots showed taproot RSC was consistently below the 5th percentile of lateral RSC. In all four sample replicates change of lateral root RSC over the drying period was 


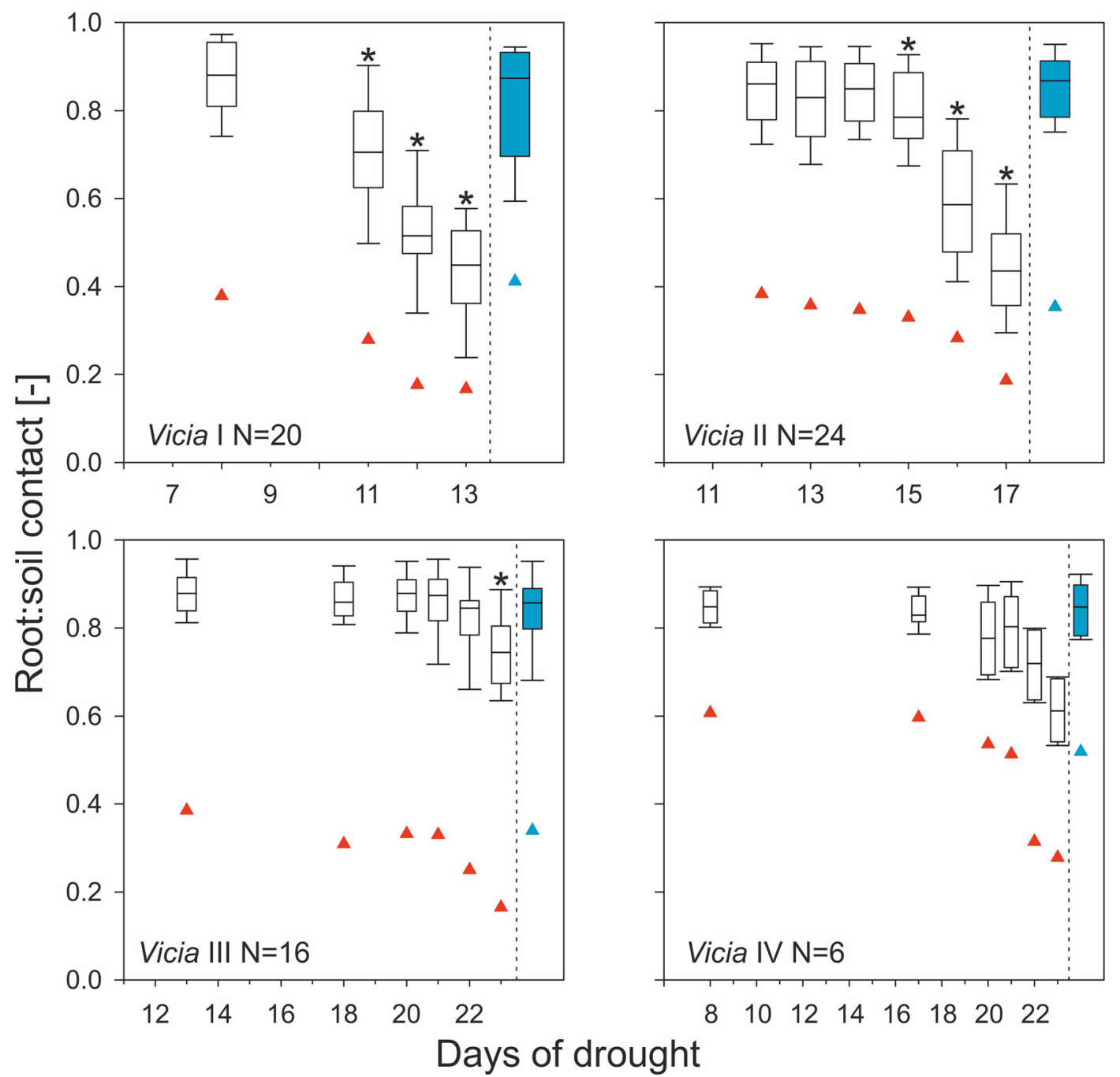

Fig. 7 Boxplots of root soil contact (RSC) during the drying period and after rewatering. Boxplots show the range of lateral root RSC for each repeated measurement, closed triangles show taproot RSC. The dashed vertical line indicates rewatering of the

statistically significant (Friedman's test, $p<0.05$ ). Comparison of individual repeated scans with the respective rewatered control showed that the temporal dynamics of shrinkage were highly variable between the plant replicates (Fig. 7). While in Vicia I, lateral RSC was significantly reduced after $11 \mathrm{~d}$ of drought treatment, in Vicia III a significantly reduced lateral RSC was not observed until $23 \mathrm{~d}$ of drought. In Vicia IV, none of the individual scans showed a statistically significant reduction of lateral RSC, probably due to the low number of replicate lateral roots. While over time, the different sample replicates behaved quite differently, Kruskal-Wallis test showed that lateral RSC of rewatered controls was not plants, rewatered controls are coloured in blue. Asterisks indicate statistically significant reduction of RSC as compared with controls (Bonferroni adjusted $p<0.0125$ in Vicia I, and $p<0.008$ in Vicia II-IV)

significantly different between the replicates $\left(\chi^{2}=\right.$ $0.277, p=0.964)$. The temporal differences are cancelled out when plotting RSC over soil matric potential so that the dependence of RSC on soil water status becomes more apparent (Fig. 8). Lateral RSC started to decrease at matric potentials below $-20 \mathrm{kPa}$, while there was still some variability between individual plant replicates. Taproot RSC was consistently lower and more variable between replicates.

A further difference between taproot and lateral roots was the location of the air gap. When the gap was at maximum extension, it formed an almost complete, concentric ring around the taproot. For 
Fig. 8 Root-soil contact (RSC) plotted over matric potential. Red symbols show lateral root RSC, blue symbols show taproot RSC. Errors are standard deviation of the mean. Different symbol shapes depict individual replicates. The dashed line on the right hand side indicates that no values for matric potential are available for the final scans. The last recorded value was for both replicates $-55 \mathrm{kPa}$. RSC after rewatering is not shown

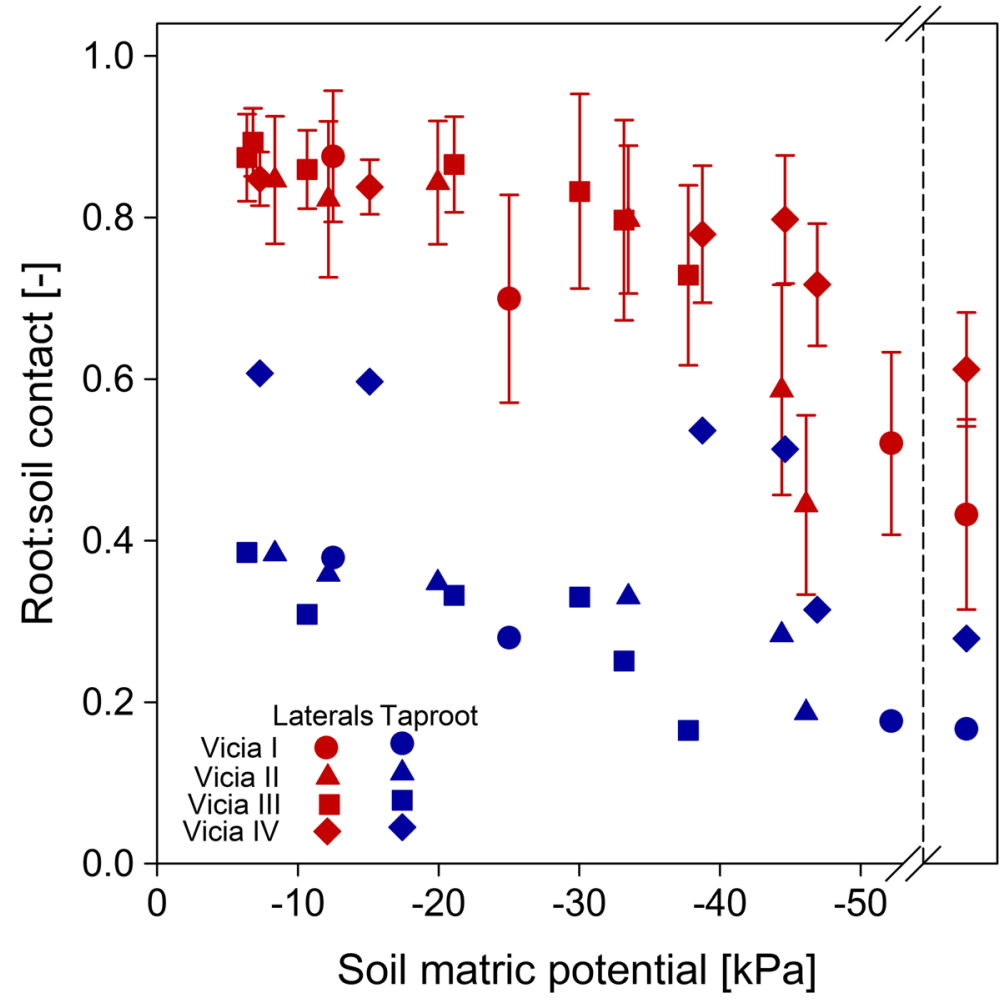

lateral roots complete contact loss was only seen at the base, while in more distal parts the air gap was eccentrically located on one side of the root, while the opposite side remained in contact with the soil.
However, in some instances there were soil particles stuck at the root surface and air filled gaps were forming few micrometers away from the immediate soil-root interface (Fig. 9b).

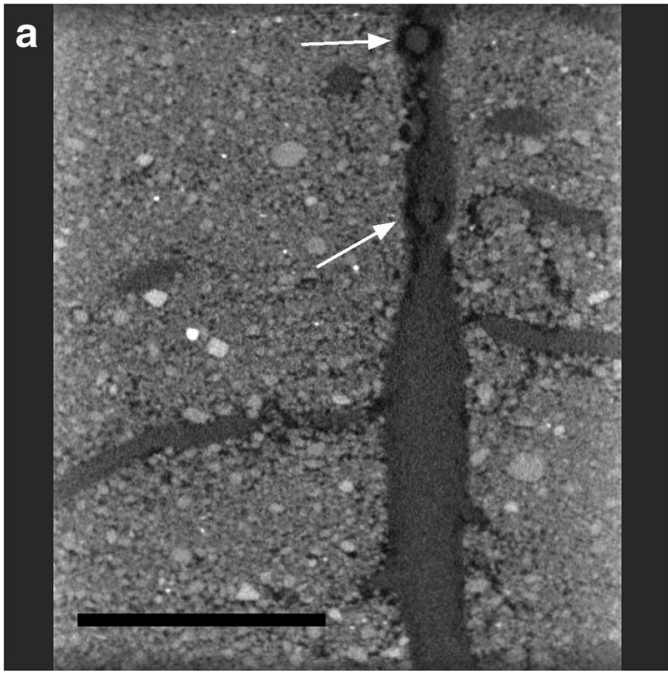

Fig. 9 a Vertical slice of Vicia I, after 8 days of the drying period. Arrows show air rings at the base of lateral roots b: Horizontal slice of Vicia II, after 17 days of the drying period. The arrow points to a gap appearing behind particles adhering to the root

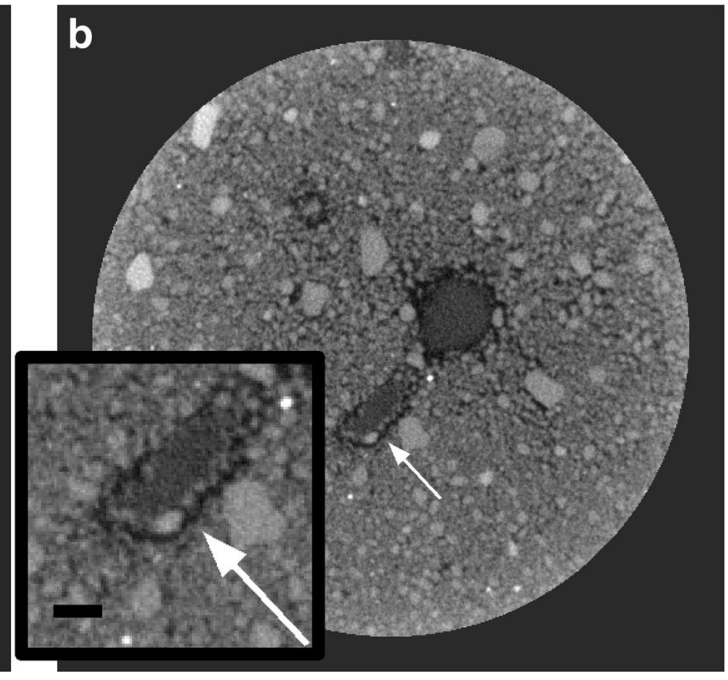

surface. The inset shows the same slice at higher magnification. The thickness of the layer adhering to the roots is ca. 5 pixels $(300 \mu \mathrm{m})$. Scalebar on the left is $10 \mathrm{~mm}$, scalebar in the inset is $1 \mathrm{~mm}$ 


\section{Discussion}

\section{Determination of RSC}

The direct method to determine RSC showed good agreement with the dilation method. The latter is similar to the procedure used by Schmidt et al. (2012), who determined that the accuracy of their method in measuring the contact area of defined phantoms was $3 \%$. The accuracy of any method is obviously affected by image quality, most notably resolution, signal to noise ratio and contrast. The threshold to segment roots from air is a particularly sensitive parameter, as our sensitivity analysis for $\mathrm{T}_{\text {air }}$ has shown. Assuming an accuracy of $10 \%$ in finding the "true" threshold, an error of $11.5 \%$ in RSC was determined. This highlights the need for an automated threshold detection to avoid user bias, as was done in this study. The effect of changing $T_{\text {root }}$ was not tested, as it is a less sensitive parameter than $\mathrm{T}_{\text {air }}$. The contrast between roots and soil matrix is much higher than the contrast between roots and air. Changing $T_{\text {root }}$ would lead to a change of the total volume of roots within the region of interest. It would therefore affect relative shrinkage, but it would not affect RSC.

At the initial matric potential of $-5 \mathrm{kPa}$, the mean diameter of drained pores calculated with the YoungLaplace equation will be approximately $60 \mu \mathrm{m}$, which is below the resolution of the scans (approx. $120 \mu \mathrm{m}$ ). In other words, a sizeable fraction of unresolved pore space might already be drained and actual root soil contact will be lower than what was measured. This is a limitation that results from the cone beam geometry. ROI scanning was used as a compromise between resolution and to reduce the effect of pot size on root architecture. While ROI scanning increases resolution it has also some disadvantages like the reduction of contrast caused by hardening of the beam and the occurrence of cupping artefacts at the truncation edges of the resulting images.

\section{Relation between root shrinkage and transpiration}

Root shrinkage was observed long after stomatal conductance decreased, showing that air gaps were not the cause of water stress. The early decrease of stomatal conductance and relative transpiration at relatively high soil matric potentials is explained by the coarse texture of the soil substrate and the resulting drop in soil hydraulic conductivity. This is in agreement with previous work on Lupinus using the same substrate (Carminati et al. 2013). Stomatal conductance was measured on the youngest unfolded leaves, which explains the steeper reduction of stomatal conductance compared to relative transpiration rate. As the responsiveness of stomata to water stress tends to decrease with leaf age (Blackman and Davies 1984; Willmer et al. 1988) it was hypothesized that stomata in older leaves retained higher aperture at moderate stress levels and closed when stress was more severe. This was confirmed by single measurements on older leaves (data not shown). When roots started to shrink at matric potentials below $-30 \mathrm{kPa}$ no further decrease of stomatal conductance of the young leaves could be observed, yet, relative transpiration rate was still decreasing. It can therefore not be excluded that the additional resistance to water flow caused by reduced RSC contributed to water stress at this point. It can again only be stated that gap formation was clearly not the initial cause of stress, confirming the conceptual model postulated in Carminati et al. (2013).

\section{Dynamics of root shrinkage}

Despite differences in growth dynamics, normalisation of RSC to soil matric potential showed that all values of lateral roots collapse onto one curve (Fig. 8), clearly demonstrating that soil water availability is the main driver of root shrinkage. The remaining variability between individual replicates can be explained by differences in plant size and duration of the drying cycle: Vicia I and II, which were overall bigger, had higher transpiration rates than the remaining samples. Vicia I had a maximum transpiration rate of $28.5 \mathrm{~g}$ water per day and plant, Vicia II $19.5 \mathrm{~g} \mathrm{~d}^{-1}$, and Vicia III and IV transpired $17 \mathrm{~g} \mathrm{~d}^{-1}$ on maximum. Vicia I and II also had the highest degree of root shrinkage, which can be explained by a higher transpirational demand. As soil matric potential was measured in the bulk soil only, the matric potential drop across the rhizosphere was not seen by the tensiometers. In Vicia I, which dried the soil faster, this drop was potentially larger because transpirational demand was higher and there was less time to equilibrate between rhizosphere and bulk soil. The differences in plant age of the individual replicates are another potential source of variability in RSC, as rhizosphere hydraulic properties can show considerable plasticity with root age (Carminati and Vetterlein 2013).

The comparison of our present results for Vicia faba with those of Carminati et al. (2013) for Lupinus albus shows that roots of both Lupinus and Vicia started to 
shrink at a similar soil matric potential between -10 to $-30 \mathrm{kPa}$. In the present study the dynamics of gap formation can be seen more accurately due to technical improvements. Our results show that the shrinkage rate increased with the duration of the drying cycle and with decreasing soil matric potential. The drying period was stopped somewhat arbitrarily when gaps were clearly visible to observe the behavior upon re-watering. Further drying would likely have resulted in a typical s-shaped pressure-volume curve as in Cole and Alston (1974). It is apparent that the soil matric potentials measured here and in Carminati et al. (2013) are much higher than the root water potentials at which Cole and Alston observed root shrinkage (between -0.5 and $-1 \mathrm{MPa}$ ), but also much higher than the soil water potentials at which Huck et al. (1970) observed diameter changes in cotton (between -0.1 and $-0.7 \mathrm{MPa})$. Even more negative soil water potentials were reported by North and Nobel (1997a, 1997b) for desert succulents, but these values were measured when gaps were already present for a long time. Again, this is explained by the coarse texture of the soil substrate. The soil matric potentials were measured in bulk soil, no measurements of either root water potential or matric potential at the soil-root interface were available. While potential gradients at the soil-root interface are difficult to measure, simulation studies show that they have a high impact on the occurrence of water stress (Schröder et al. 2009) and that they are more important in coarse textured soils (Javaux et al. 2008). When roots start to shrink and the hydraulic pathway is reduced by decreasing RSC, the gradient at the root-soil interface will become even larger. Even when roots retain partial contact, flux density will increase at a given volumetric flow rate leading to a larger water potential drop (Faiz and Weatherley 1982; Tinker 1976).

\section{Taproots versus laterals}

The most striking difference between Vicia and experiments with Lupinus in Carminati et al. (2013) was the different behavior of taproots and laterals. While in Lupinus the taproot shrank more than laterals, in roots of Vicia no significant difference of relative shrinkage between taproots and lateral roots was found. A major difference between both species is the thickness of lateral roots. While Vicia laterals in the present study had a diameter of over $1000 \mu \mathrm{m}$, Lupinus lateral roots were generally below $500 \mu \mathrm{m}$ in diameter. If gap size is calculated for these diameters, relative shrinkage of
$10 \%$ would result in a gap of 50-100 $\mu \mathrm{m}$ (depending on the eccentricity) in Vicia and 25-50 $\mu \mathrm{m}$ in Lupinus. Consequently, gaps around Vicia roots are easier to detect. Carminati et al. admitted that their spatial resolution $(100 \mu \mathrm{m}$ voxel size) was limiting the detection of gaps around laterals. They suggested the use of a higher resolution to confirm these results, which was done in the present study. As they have rightly pointed out, bigger gaps will be drained at higher matric potentials, meaning that thicker roots lose hydraulic contact earlier. The possibility that there are other anatomical or physiological differences between the roots of both species, which further explain the observed differences cannot be excluded. Additionally, significant variations of root traits can be observed between different genotypes of one species, i.e. the differences that were found cannot be interpreted as a species effect per se.

Different resolution can certainly not explain the observed difference of taproot shrinkage. Taproots in the present study had only partial contact with the soil even under well-watered conditions. It would seem likely that the potential drop at their interface would therefore be larger and shrinkage would be more pronounced, as was suggested by Carminati et al. (2013). A possible explanation why there was no difference in shrinkage of taproots and laterals despite larger diameter of the taproot is a higher radial resistance to water flow due to the suberization of the taproot endodermis. This would isolate the xylem and reduce water depletion from the cortex. Typically, radial resistance of older roots tends to increase with the development of apoplastic barriers (Bramley et al. 2009; Frensch and Steudle 1989). As a consequence, RSC of the older taproot is less critical. This is in agreement with the notion that the main function of taproots or primary roots is long distance transport, while laterals are the main sites of water and nutrient uptake (McCully 1999). An alternative explanation is that taproots of Vicia underwent more secondary growth than Lupinus, resulting in a larger share of the rigid stele versus cortex area.

\section{RSC of the taproot - The role of lateral emergence}

Initial restriction of RSC of taproots had not been reported by Carminati et al. (2013), as the authors did not quantify RSC, but width of the air gap and diameter of the roots, respectively. In the present study, low contact of the taproot coincided with the emergence of laterals. In many instances there was an obvious ring of air 
around the laterals, exactly where they emerged from the taproot (Fig. 9a). As laterals emerge they have to rupture the cortex. This is beautifully illustrated in Pond's drawing of a seedling (Pond 1908), where the ruptures closely resemble the rings of air that were observed. As laterals advance further they displace soil particles in their way, thereby increasing the size of these localized air gaps. A further cause of lower RSC at the taproot could be the lower abundance of root hairs and mucilage, which are both thought to be critical for the establishment of good RSC.

Lateral roots retain partial contact

Finally, it is observed that lateral roots retained partial contact with the soil, although they exhibited higher relative shrinkage than taproots. It cannot be stated with certainty, that partial contact with the soil would have been maintained during further drying. However, referring to the introduction, it is more likely that an additional contraction of the roots will lead to more shrinkage on the side where the surface is no longer in contact with the soil. Partial contact might even be crucial to maintain water and nutrient uptake under drought conditions, as elegant experiments have shown that reestablishment of RSC by "squeezing" or vibrating the soil temporarily reduced water stress in Helianthus annuus (Faiz and Weatherley 1982). While the squeezing treatment might have resulted in a change of the water retention curve, which could partly explain these results, other work has shown that water and nitrate uptake decreases with decreasing RSC (Veen et al. 1992). In roots growing into pre-existing macropores, partial contact may even determine patterning of lateral roots. Bao et al. (2014) showed that lateral root production of roots with partial contact at one side was biased towards the contact side. This bias was not caused by contact per se but by the higher water availability at the contact side.

\section{Conclusions \& Outlook}

An efficient method of measuring the dynamics of root shrinkage and RSC in a drying soil using X-ray CT scans of Vicia faba roots grown in a sandy soil substrate was presented. The study confirms previous work with Lupinus albus in that root shrinkage and the formation of air gaps at the root-soil interface occurs when transpiration rate is already severely reduced. Both Vicia and Lupinus showed a similar reaction to water stress and root shrinkage was initiated at similar soil matric potentials. We therefore confirm that root shrinkage is a consequence and not the cause of water stress. When gaps appear, they will contribute to water stress by narrowing the hydraulic pathways and increasing the water potential drop at the root-soil interface.

A major difference between the analyzed genotypes of both species was the extent of shrinkage in the different root orders. While in Lupinus lateral roots shrank less than taproots, the present study shows that in Vicia no significant difference was found between taproots and laterals. Whether the differences are merely a result from different image resolution or there are true anatomical and physiological differences remains uncertain. The different dynamics of shrinkage between individual replicates show that the degree of shrinkage varies with the size and the transpiration rate of individual plants, i.e. bigger plants with higher transpiration rates are affected more. It is concluded that the dynamics of root shrinkage are governed by soil water availability and transpirational demand.

Clearly, more comparative studies are needed to answer this question, preferably involving more species from different plant families. It was shown that lateral roots retain partial contact with the soil, thus further enabling the uptake of nutrients and water. Retention of partial contact may be related to the abundance of root hairs and mucilage, but further studies involving plants differing in those traits are needed to confirm this.

As root shrinkage is closely related to root water potential, diurnal variation of RSC is expected. Future studies should therefore include pre-dawn measurements to see if gaps close completely at night with the relaxation of plant water potential.

Acknowledgments NK was funded by Helmholtz Impulse and Networking Fund through Helmholtz Interdisciplinary Graduate School for Environmental Research (HIGRADE).

Open Access This article is distributed under the terms of the Creative Commons Attribution 4.0 International License (http:// creativecommons.org/licenses/by/4.0/), which permits unrestricted use, distribution, and reproduction in any medium, provided you give appropriate credit to the original author(s) and the source, provide a link to the Creative Commons license, and indicate if changes were made. 


\section{References}

Bao Y, Aggarwal P, Robbins NE, Sturrock CJ, Thompson MC, Tan HQ, Tham C, Duan L, Rodriguez PL, Vernoux T, Mooney SJ, Bennett MJ, Dinneny JR (2014) Plant roots use a patterning mechanism to position lateral root branches toward available water. Proc Natl Acad Sci 111:9319-9324

Bernstein L, Gardner WR, Richards LA (1959) Is there a vapor gap around plant roots. Science 129:1750-1753

Blackman PG, Davies WJ (1984) Age-related changes in stomatal response to Cytokinins and abscisic acid. Ann Bot 54:121126

Bramley H, Turner NC, Turner DW, Tyerman SD (2009) Roles of morphology, anatomy, and Aquaporins in determining contrasting hydraulic behavior of roots. Plant Physiol 150:348364

Bruand A, Cousin I, Nicoullaud B, Duval O, Begon JC (1996) Backscattered electron scanning images of soil porosity for analyzing soil compaction around roots. Soil Sci Soc Am J 60:895-901

Carminati A, Vetterlein D, Koebernick N, Blaser S, Weller U, Vogel HJ (2013) Do roots mind the gap? Plant Soil 367: 651-661

Carminati A, Vetterlein D, Weller U, Vogel HJ, Oswald SE (2009) When roots lose contact. Vadose Zone J 8:805-809

Carminati A, Vetterlein D (2013) Plasticity of rhizosphere hydraulic properties as a key for efficient utilization of scarce resources. Ann Bot 112:277-290

Cole PJ, Alston AM (1974) Effect of transient dehydration on absorption of chloride by wheat roots. Plant Soil 40:243-247

Dexter AR (1987) Compression of soil around roots. Plant Soil 97: 401-406

Faiz SMA, Weatherley PE (1982) Root contraction in transpiring plants. New Phytol 92:333-343

Frensch J, Steudle E (1989) Axial and radial hydraulic resistance to roots of maize (Zea mays L.). Plant Physiol 91:719-726

Haling RE, Tighe MK, Flavel RJ, Young IM (2013) Application of $\mathrm{X}$-ray computed tomography to quantify fresh root decomposition in situ. Plant Soil 372:619-627

Hettiaratchi DRP, Goss MJ, Harris JA, Nye PH, Smith KA (1990) Soil compaction and plant root growth. Philos Trans R Soc Lond B Biol Sci 329:343-355

Herkelrath WN, Miller EE, Gardner WR (1977) Water-uptake by plants. 2. Root contact model. Soil Sci Soc Am J 41:10391043

Huck MG, Klepper B, Taylor HM (1970) Diurnal variations in root diameter. Plant Physiol 45:529-530

Javaux M, Schroder T, Vanderborght J, Vereecken H (2008) Use of a three-dimensional detailed modeling approach for predicting root water uptake. Vadose Zone J 7:1079-1088

Kooistra MJ, Schoonderbeek D, Boone FR, Veen BW, Vannoordwijk M (1992) Root-soil contact of maize, as measured by a thin-section technique. 2. Effects of soil compaction. Plant Soil 139:119-129

Leitner D, Klepsch S, Ptashnyk M, Marchant A, Kirk GJD, Schnepf A, Roose T (2010) A dynamic model of nutrient uptake by root hairs. New Phytol 185:792-802

MacFall JS, Johnson GA, Kramer PJ (1991) Comparative water uptake by roots of different ages in seedlings of loblolly pine (Pinus taeda L.). New Phytol 119:551-560
McCully ME (1999) Roots in soil: unearthing the complexities of roots and their rhizospheres. Annu Rev Plant Biol 50:695718

McGinnis R (2002-2009) Rad pro X-ray device dose-rate calculator online. Massachusetts Institute of Technology

Mooney SJ, Pridmore TP, Helliwell J, Bennett MJ (2012) Developing X-ray computed tomography to non-invasively image 3-D root systems architecture in soil. Plant Soil 352:1-22

North GB, Nobel PS (1997a) Root-soil contact for the desert succulent Agave deserti in wet and drying soil. New Phytol 135:21-29

North GB, Nobel PS (1997b) Drought-induced changes in soil contact and hydraulic conductivity for roots of Opuntia ficusindica with and without rhizosheaths. Plant Soil 191:249-258

Nye PH (1994) The effect of root shrinkage on soil-water inflow. Philos Trans R Soc Lond Ser B Biol Sci 345:395-402

Philip JR (1957) The physical principles of soil water movement during the irrigation cycle. Proc. Int. Congr. Comm. Irrig. Drain. , 3rd, San Francisco 8:125-154

Pond RH (1908) Emergence of lateral roots. Bot Gaz 46:410-421

Rosin PL (2001) Unimodal thresholding. Pattern Recogn 34: 2083-2096

Schlüter S, Sheppard A, Brown K, Wildenschild D (2014) Image processing of multiphase images obtained via X-ray microtomography: a review. Water Resour Res 50:36153639

Schmidt S, Bengough AG, Gregory PJ, Grinev DV, Otten W (2012) Estimating root-soil contact from 3D X-ray microtomographs. Eur J Soil Sci 63:776-786

Schröder T, Javaux M, Vanderborght J, Körfgen B, Vereecken H (2009) Implementation of a microscopic soil-root hydraulic conductivity drop function in a three-dimensional soil-root architecture water transfer model. Vadose Zone J 8:783-792

Tinker PB (1976) Transport of water to plant roots in soil. Philos Trans R Soc Lond B Biol Sci 273:445-461

Van Noordwijk M, Kooistra MJ, Boone FR, Veen BW, Schoonderbeek D (1992) Root-soil contact of maize, as measured by a thin-section technique. Plant Soil 139:109-118

Veen BW, Van Noordwijk M, De Willigen P, Boone FR, Kooistra MJ (1992) Root-soil contact of maize, as measured by a thinsection technique. Plant Soil 139:131-138

Vetterlein D, Marschner H, Horn R (1993) Microtensiometer technique for Insitu measurement of soil matric potential and root water extraction from a Sandy soil. Plant Soil 149: 263-273

Vogel HJ, Weller U, Schlüter S (2010) Quantification of soil structure based on Minkowski functions. Comput Geosci 36:1236-1245

Vollsnes AV, Futsaether CM, Bengough AG (2010) Quantifying rhizosphere particle movement around mutant maize roots using time-lapse imaging and particle image velocimetry. Eur J Soil Sci 61:926-939

Willmer CM, Wilson AB, Jones HG (1988) Changing responses of stomata to abscisic acid and $\mathrm{CO} 2$ as leaves and plants age. J Exp Bot 39:401-410

Zappala S, Helliwell JR, Tracy SR, Mairhofer S, Sturrock CJ, Pridmore T, Bennett M, Mooney SJ (2013) Effects of XRay Dose On Rhizosphere Studies Using X-Ray Computed Tomography PLoS ONE:8 